\title{
Study the Effect of Added 4wt\% of Nickel Oxide, Ammonium Oxide and Cobalt Oxide over HZSM-5 Catalyst for the Catalytic reaction of Bio-Ethanol into Hydrocarbons
}

\author{
Thamer Adnan Abdullah ${ }^{1}$, Fanar Ganem Hashem ${ }^{1}$, Qusay J. Rasheed ${ }^{2}$, Abdulnasser M. AL-gebori ${ }^{1}$, \\ Hasan R. Obayes ${ }^{1}$ \\ ${ }^{1}$ Applied Sciences Department/Applied Chemistry Branch, University of Technology, Baghdad, Iraq \\ ${ }^{2}$ Department of Production Engineering and Metallurgy/Mining and Metal Extractive Engineering Branch, \\ University of Technology, Baghdad, Iraq
}

\begin{abstract}
Conversion of Bio-Ethanol into hydrocarbons has been studied on Zeolite HZSM-5, 4wt\% Nickel Oxide/HZSM-5, 4wt\% Ammonium Oxide/HZSM-5 and 4wt\% Cobalt Oxide/HZSM-5. All the modified zeolite catalyst are tested by conducted in fixed bed reactor at $450^{\circ} \mathrm{C}$, pressure $=1$ atm and $W H S V=2.5 \mathrm{~h}^{-1}$. It has been recognized that 4wt\% Cobalt Oxide/HZSM-5 has highly resistance to coke formation over catalyst. It was observed 4wt\% Nickel Oxide /HZSM-5 and 4wt\% Ammonium Oxide /HZSM-5 loose activity faster as compared to 4wt\% Cobalt Oxide/HZSM-5. Prepared and modified ZSM-5 catalysts were characterized by XRD, BET and $T G A$, as well as burning in muffle furnace after experimental runs. Experimental results were shown that Cobalt Oxide modified HZSM-5 improved catalytic performance and enhanced catalyst life.
\end{abstract}

Keywords: HZSM-5, Coke formation, Bio-ethanol conversion, Catalyst Characterization

\section{Introduction}

Conversion of bioethanol into hydrocarbons has raised considerable interest in scientific community in the last years, particularly since the price of fossil fuels increased and environmental policies became stricter. Bioethanol transformation to hydrocarbons can be used as fuel in transportation. Light olefins such as ethylene, propylene are important intermediate and building blocks in petrochemicals industry; ethylene is the most widely produce in chemical industry. HZSM-5 zeolite was developed by the time 1970 at this time of oil crises the synthesis gasoline because of break demand of hydrocarbons was found to be promising. Preparation of HZSM-5 catalyst by 4 wt\% nickel oxide, ammonium oxide and cobalt oxide increases the conversion and product yield of hydrocarbons particularly light olefins. Using impregnation method technique ZSM-5 catalyst has been modified by loading with different weight percent of metals [1-6].

Catalytic dehydration of ethanol to ethylene and ethanol to aromatics are some of studies in this direction [14]. Conversion of ethanol to hydrocarbons over zeolite has been studied due to increase demand of hydrocarbons in the market [5-14]. Objective of this work is to investigate the influence of nickel oxide, ammonium oxide and cobalt oxide loading on catalytic performance of HZSM-5.

In this study the important of nickel oxide, ammonium oxide and cobalt oxide doping HZSM-5 catalyst has been studied for the selective conversion of ethanol and hydrocarbon yield. Effect of nickel oxide, ammonium oxide and cobalt oxide doping over HZSM-5 catalyst was also investigated the activity and selectivity of HZSM5 catalyst for ethanol into hydrocarbons process. The catalysts were characterized by different technique including BET surface area analyzer, X-ray diffraction (XRD) and thermos gravimetric analysis (TGA).

\section{Catalyst Modification}

HZSM-5 was used as support for nickel oxide, ammonium oxide and cobalt oxide, catalyst was prepared by impregnation method technique with 4 wt\%, nickel oxide, ammonium oxide and cobalt oxide doped HZSM-5 catalyst and the final solution was stirred for $11 \mathrm{~h}$ at $308 \mathrm{~K}$, and the final slurry was transferred to rotary evaporator to remove excess water under vacuum at $343 \mathrm{~K}$, followed by drying in an oven at $383 \mathrm{~K}$ for $8 \mathrm{~h}$. The dried pellet was calcined in the furnace at $823 \mathrm{~K}$ for $5 \mathrm{~h}$. 


\section{Catalyst characterization}

The prepared catalysts were characterized by X-ray diffraction (XRD), Surface area and pore size analyzer and TGA was used to analyze weight loss during heat treatment and to estimate the coke deposited on the catalyst surface after reaction

\section{Experimental Work}

Zeolite HZSM-5 is the catalyst used for ethanol to hydrocarbon conversion; it was used as a support for nickel oxide, ammonium oxide and cobalt oxide respectively. Catalyst was prepared by doping separately $4 \mathrm{wt} \%$ ammonium oxide, 4wt \% cobalt oxide and cobalt oxide onto zeolite HZSM-5 prepared by impregnation method. The final solution was stirred for $11 \mathrm{~h}$ at $308 \mathrm{~K}$. The final catalyst slurry was transferred to a rotary evaporator to remove excess water under vacuum at $343 \mathrm{~K}$ followed by drying in an oven at $383 \mathrm{~K}$ for $11 \mathrm{~h}$. The pellet catalyst which dried was calcined in a furnace at $833 \mathrm{~K}$ for $8 \mathrm{~h}$ to decompose cobalt ammonium, nickel complex to nickel oxide, ammonium oxide and cobalt oxide.

The prepared catalysts were characterized by X-ray diffraction (XRD), Surface area and pore size analyzer, Metal trace analyzer and TGA as well as burning sample from used and fresh catalyst to see the deactivation of zeolite catalyst. The surface areas and pore volumes of the catalysts were determined by using BET surface area analyzer by adsorption with nitrogen $(99.99 \%$ purity) at $77 \mathrm{~K}$, employing the static volumetric technique. The samples were placed in the evacuated sample tube, then cooled to cryogenic temperature and then exposed to the analysis gas at controlled pressures. With each incremental pressure, a gas molecules number adsorbed on the surface increases. The pressure at which adsorption equilibrium occurs was measured and quantity of gas adsorbed was determined.

ETH reaction was conducted at $450{ }^{\circ} \mathrm{C}$ in fixed bed reactor using $2 \mathrm{gm}$ of the catalyst. All the HZSM-5 catalysts were compared for their performance for ethanol conversion by conducting experiments under identical conditions. The reaction temperature was measured by $\mathrm{K}$ type thermocouple inserted in the reactor by thermocouple. Prior to reaction, the sample of $1.5 \mathrm{~g}$ were pretreated in nitrogen at $400^{\circ} \mathrm{C}$ for $2 \mathrm{~h}$. Liquid ethanol was fed and evaporated by preheater at $200^{\circ} \mathrm{C}$ to reactor by a precipitating pump and vaporized ethanol was made to enter in reactor at WHSV $=2.5 \mathrm{~h}^{-1}$. The reactor products was analyzed by gas chromatographs: flame ionization detector and thermal conductivity detector (FID and TCD).

TGA was used to determine the loss of weight during heat treatment and also estimate the coke deposited on the catalyst surface after reaction. The catalyst samples were crushed to fine powder and then transferred to a microbalance placed inside the temperature programmable furnace. The variation in mass of the catalyst was determined with the increase in temperature, due to burning of coke deposit over HZSM-5 catalyst.

Another experiment has been done to determine coke deposit over HZS-5 catalyst, was burning the catalyst used samples in muffle furnace to burn the formed coke over HZSM-5 catalyst after $20 \mathrm{~h}$ run.

\section{Result and Discussion}

The surface area and pore volume of the catalyst were evaluated by BET surface area technique. The surface area of catalyst decreases on loading of cobalt oxide, nickel oxide and ammonium oxide. The surface area and pore volume of the catalyst were determined by BET surface area technique. The surface area of catalyst decreases on doping of metal oxide. Volume of $\mathrm{N}_{2}$ adsorbed decreased with $4 \mathrm{wt} \%$ of cobalt oxide, $4 \mathrm{wt} \%$ nickel oxide and $4 \mathrm{wt} \%$ ammonium oxide due to partial coverage of the surface with these metal oxides. It was observed that ammonium oxide, cobalt oxide and nickel oxide may enter into the channels. During experimental run it was found that coke filled the pores of HZSM-5 catalyst because of there is decrease in the pore volume and surface area of catalyst.

Crystalline phase is present in ammonium oxide, cobalt oxide and nickel oxide HZSM-5 catalyst. In crystalline phase no any new species were observed by XRD in the metal oxides doped HZSM-5. The intensity of metal oxide doped catalyst had slightly decreased. It was observed that the overall structure of catalysts remained almost same with no clear change. 


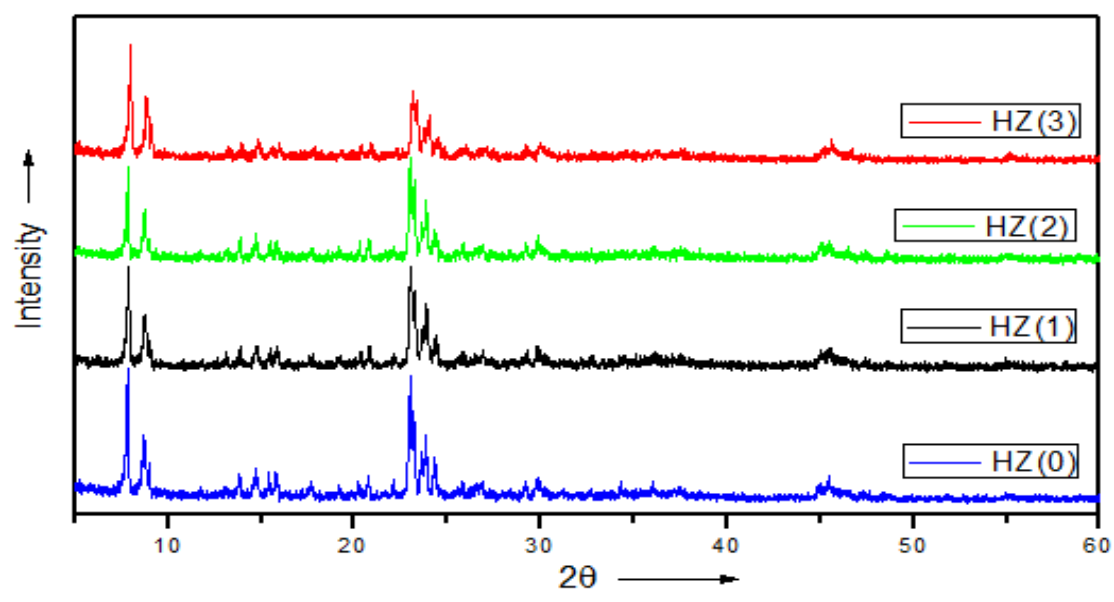

Fig. 1: XRD patterns of HZ (0): HZSM-5, HZ (1): 4wt\%cobalt oxide-HZSM-5, HZ (2): 4wt\%nickel oxide-HZSM-5, HZ (3): 4wt\% ammonium oxide-HZSM-5

The TG analysis was performed to study the coke deposition on, HZ (0) and HZ (1) catalyst after a run of 20 $\mathrm{h}$ and $\mathrm{HZ}(0)$ is taken as a (fresh catalyst). Experimental TGA results was shown that the total weight loss up to $700{ }^{\circ} \mathrm{C}$ was shown $4.3 \mathrm{wt} \%$ lose, $8.7 \mathrm{wt} \%, 9.31 \mathrm{wt} \%$ lose and $10.20 \mathrm{wt} \%$ loss for HZ (0), HZ (1), HZ (2) and HZ (3) respectively. The weight loss below $200{ }^{\circ} \mathrm{C}$ is mainly due to the physically adsorbed water in the porous material. The weight loss in case of $\mathrm{HZ}$ (1) catalyst is less, which means the amount of coke deposition over $\mathrm{HZ}$ (1): (4wt\%cobalt oxide-HZSM-5) is less as compare with HZ (0), HZ (2) and HZ (3).

Table1 shows the loss of the weight of catalyst samples after $20 \mathrm{~h}$ run by burning in muffle furnace at $550{ }^{\circ} \mathrm{C}$ for $3 \mathrm{~h}$ to burn coke deposit at the surface of HZSM-5 catalyst. The used catalyst samples after $20 \mathrm{~h}$ run was transferred to oven at $110{ }^{\circ} \mathrm{C}$ to remove moisture. Then $0.20 \mathrm{gm}$ from each sample has been taken and burn in muffle furnace to remove coke which deposit over HZSM-5 catalyst. It was shown the loss in weight was less in case of HZ (1): 4wt\% cobalt oxide modified HZSM-5 catalyst, that means HZ (1) catalyst had high resistance to coke formation and enhanced HZSM-5 catalyst.

TABLE I: Burning used samples and remove coke deposition. HZ (0): HZSM-5, HZ (1): 4wt\%cobalt oxide-HZSM-5, HZ(2): 4wt\%nickel oxide-HZSM-5, HZ (3): 4wt\% ammonium oxide-HZSM-5

\begin{tabular}{|c|c|c|}
\hline $\begin{array}{c}\text { HZSM-5 } \\
\text { Samples }\end{array}$ & $\begin{array}{c}\text { Weight before } \\
\text { muffle furnace }\end{array}$ & $\begin{array}{c}\text { Weight after } \\
\text { muffle furnace }\end{array}$ \\
\hline $\mathrm{HZ}(1)$ & $0.200 \mathrm{gm}$ & $0.194 \mathrm{gm}$ \\
\hline $\mathrm{HZ} \mathrm{(2)}$ & $0.200 \mathrm{gm}$ & $0.191 \mathrm{gm}$ \\
\hline $\mathrm{HZ} \mathrm{(3)}$ & $0.200 \mathrm{gm}$ & $0.187 \mathrm{gm}$ \\
\hline $\mathrm{HZ}(0)$ & $0.200 \mathrm{gm}$ & $0.184 \mathrm{gm}$ \\
\hline
\end{tabular}

Effect of HZ (1) on conversion and products yield as function of time on stream has been shown in Fig. 2 and Fig. 3. Compared with HZ (0), HZ (2), HZ (3) catalysts, an increase in conversion and hydrocarbon yield was observed with HZ (1). The major products of the reaction were methane, ethylene, propylene, dimethyl ether, xylene, isopropyl benzene, toluene, ethyl benzene, trimethyl benzene and tetra methylbenzene. Fig. 2 shows a comparison of effect of run time on conversion with HZ (0), HZ (1), HZ (2) and HZ (3) catalysts.

As can be seen from Fig. 2, conversion of ethanol decreases with increase in time on stream. Initial conversion of $\mathrm{HZ} \mathrm{(0),} \mathrm{HZ} \mathrm{(1),} \mathrm{HZ} \mathrm{(2),} \mathrm{and} \mathrm{HZ} \mathrm{(3)} \mathrm{were} \mathrm{76.4 \% ,} \mathrm{98.3 \% ,} 97.81$ and $87.35 \%$ respectively and after $20 \mathrm{~h}$ run ethanol conversion decreased to $49.3 .3 \%, 79.9 \%, 68.9 \%$ and $60.75 \%$ respectively for these catalysts. 


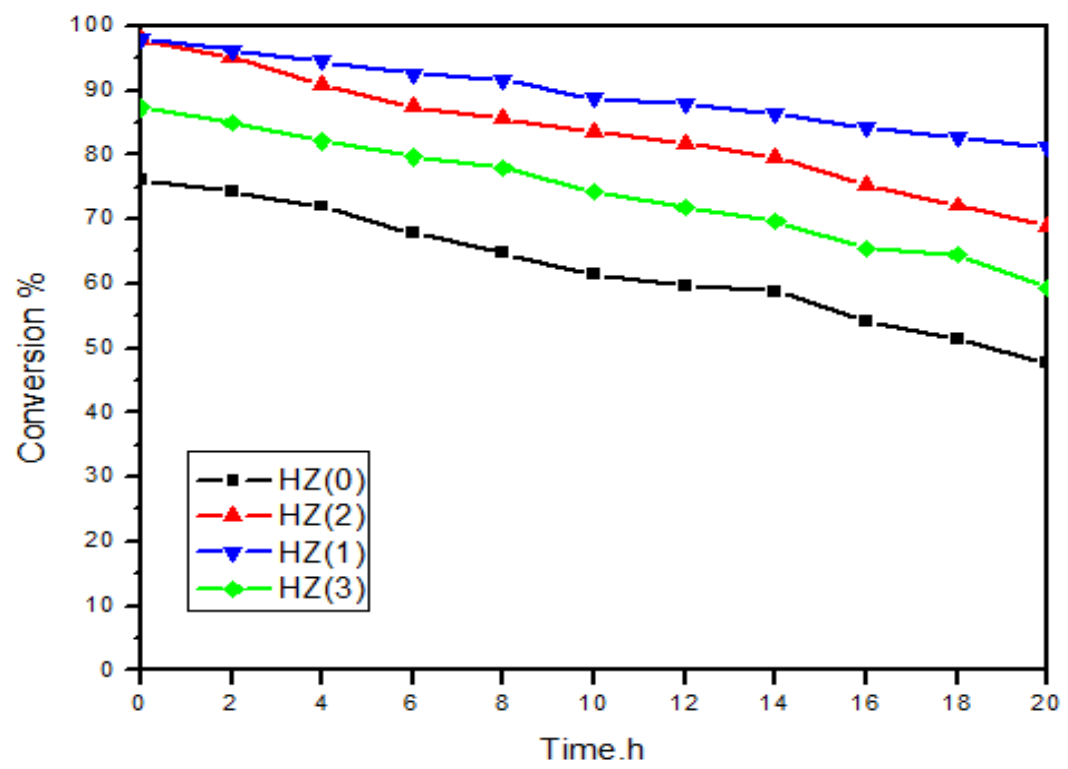

Fig. 2: Conversion of ethanol versus Time in a Fixed Bed Reactor $\left[\mathrm{T}=4500^{\circ} \mathrm{C}, \mathrm{P}=1 \mathrm{~atm}\right.$ and WHSV $\left.=2.5 \mathrm{~h}^{-1}\right]$. HZ (0): HZSM5, HZ (1): 4wt\% cobalt oxide-HZSM-5, HZ(2): 4wt\%nickel oxide-HZSM-5, HZ (3): 4wt\% ammonium oxide-HZSM-5

Fig. 3 shows the yield of hydrocarbons with respect to time. With increase in time of stream also results is decreased in the yield of hydrocarbons. Fig. 3 also shows a comparison of effect of run time on products yield with $\mathrm{HZ}(0), \mathrm{HZ}(1), \mathrm{HZ}(2)$ and HZ (3) catalysts.

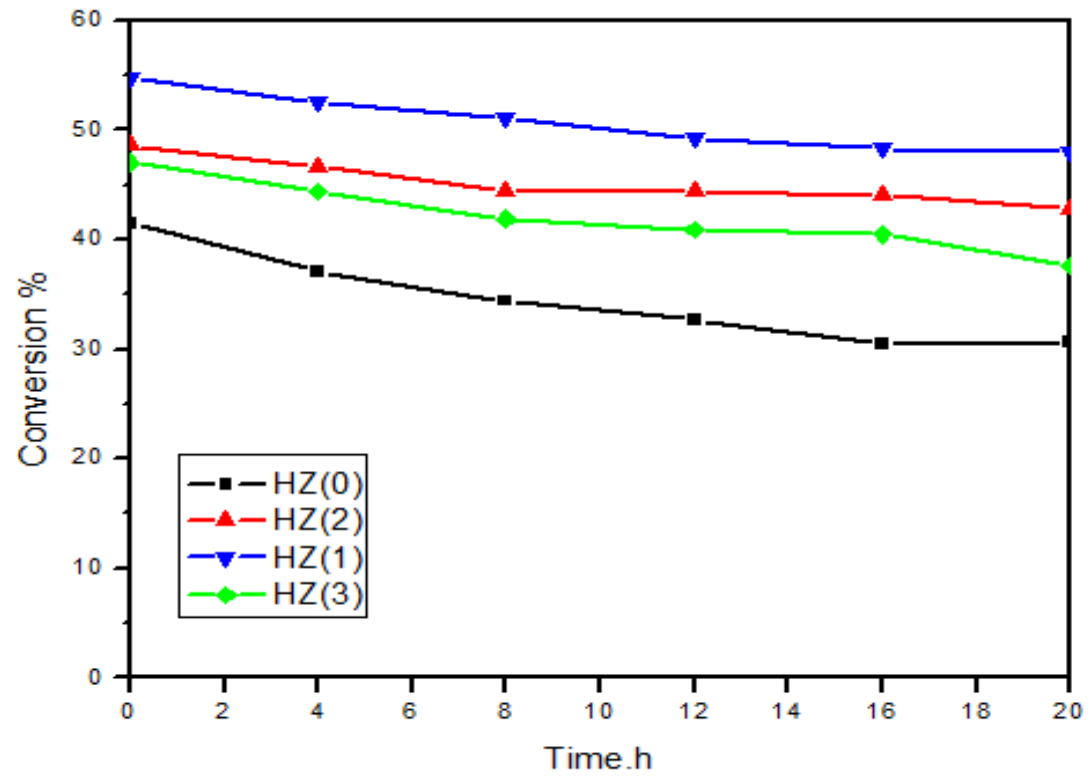

Fig. 3: Yield of Hydrocarbon versus Time in a Fixed Bed Reactor $\left[\mathrm{T}=450{ }^{\circ} \mathrm{C}, \mathrm{P}=1 \mathrm{~atm}\right.$ and $\left.\mathrm{WHSV}=2.5 \mathrm{~h}^{-1}\right] . \mathrm{HZ}(0)$ : HZSM-5, HZ (1): 4wt\%cobalt oxide-HZSM-5, HZ (2): 4wt\%nickel oxide-HZSM-5, HZ (3): 4wt\% ammonium oxideHZSM-5

As can be seen from this Fig. 3, Initial hydrocarbons yield of HZ (0), HZ (1), HZ (2) and HZ (3) were 41.5\%, $54.7 \%, 48.61 \%$ and $47.03 \%$ respectively and after $20 \mathrm{~h}$ run, hydrocarbon yield decreased to $30.7 \%, 48.1 \%$, $42.8 \%$ and $38.6 \%$ respectively. Fig2 and fig 3 shows the addition of HZ (1): (cobalt oxide-HZSM-5 catalyst) increases the active sites on HZSM-5, which are highly reactive selective to hydrocarbons and increase the conversion and yields of hydrocarbons as compare with HZ (0), HZ (2) and HZ (3). 


\section{Conclusions}

Addition 4wt\% Cobalt Oxide doped HZSM-5: HZ (1) catalyst has been recognized very high stability and selectivity to hydrocarbons products as compared with 4wt\% Nickel Oxide modified HZSM-5: HZ (2), 4wt\% Ammonium Oxide modified HZSM-5: HZ (3) and HZM-5 parent catalyst: HZ (0) in the conversion of ethanol to hydrocarbons. HZ (1) catalyst shows higher resistance to formation of coke as compared with HZ (2), HZ (3) and HZ (0) catalysts. Decrease in acidity of catalyst after doping with Cobalt Oxide lead to less coke deposit over HZSM-5 catalyst as compared to the other prepared catalysts. Cobalt oxide doped HZSM-5 catalyst, has been recognized high resistance to poison of catalyst and more hydrocarbons yield. Acknowledgments

The Authors would like to express their appreciation to the Ministry of Higher Education and Scientific Research in Iraq and University of Technology, Baghdad, Iraq for supporting this work.

\section{References}

[1] Gayubo AG, Alonso A, Valle B, Aguayo AT, Olazar M, and Bilbao J. Hydrothermal stability of HZSM-5 catalysts modified with Ni for the transformation of bioethanol into hydrocarbons. Fuel 2010; 89: 3365-3372. http://dx.doi.org/10.1016/j.fuel.2010.03.002

[2] K. Ramesh, L. M. Hui, Y. F. Han, and A. Borgn, Structure and reactivity of phosphorous modified H-ZSM-5 catalysts for ethanol dehydration. Catalysis Communications. (10). 2009, 567-571.

[3] J. Bia, M. Liua, C., X. Wanga, and Guoa, X. C2-C4 light olefins from bioethanol catalyzed by Ce-modified nanocrystalline HZSM-5 zeolite catalysts. Applied Catalysis B: Environmental (107). 2011, 68-76 .

[4] G. Carotenuto, A. Kumar, J. Miller, A. Mukasyan, and E. Santacesaria, Hydrogen production by ethanol decomposition and partial oxidation over copper/copper-chromite based catalysts. Catalysis Today, (203). 2013, 163175.

[5] J. Gurgul, R. P. Socha, and S. Dzwigaj, Effect of Cu content on the catalytic activity of Cu SiBEA zeolite in the SCR of NO by ethanol: Nature of the copper species, Applied Catalysis B: Environmental, (91). 2009, 217-224.

[6] A. Takahashi, W. Xia, I. Nakamura, H. Shimada, and T. Fujitani, Effects of added phosphorus on conversion of ethanol to propylene over ZSM-5 catalysts. Applied Catalyst A: General (423-424). 2012,. 162-167.

[7] F, F. Madeira, N. S. Gnep, P. Magnoux, S. Maury, and N. Cadran, Ethanol transformation over HFAU, HBEA and HMFI zeolites presenting similarBrønsted acidity. Applied Catalyst A: General (367). 2009, 39-46.

[8] J. J. Saceda, K. Rintramee, S. Khabuanchalad, S. Prayoonpokarach, R. L. Leon, and J. Wittayakun, Properties of zeolite $\mathrm{Y}$ in various forms and utilization as catalysts or supports for cerium oxide in ethanol oxidation. Journal of Industrial and Engineering Chemistry (18), 2012, 420-424.

[9] Y. Tavan, M. R. K. Nikou, and A. Shariati, Effect of the P/Al ratio on the performance of modified HZSM-5 form ethanol dehydration reaction. Journal of Industrial and Engineering Chemistry JIEC (1381). 2013.

[10] G. Songa, A. Takahashia, I. Nakamuraa, and T. Fujitani, Phosphorus-modified ZSM-5 for conversion of ethanol to propylene. Applied Catalyst A: General. (384). 2010, 201-205.

[11] W. Kiatkittipong, S. Wongsakulphasatch, N. Tintan, N. Laosiripojana, P. Praserthdam, and S. Assabumrungrat, Gasoline upgrading by self-etherification with ethanol on modified beta-zeolite. Fuel Processing Technology. (92). 2011, 1999-2004 .

[12] P. Rybak, B. Tomaszewska, A. Machocki, W. Grzegorczyk, and A. Denis, Conversion of ethanol over supported cobalt oxide catalysts. Catalysis Today. (176). 2011, 14-20.

[13] Q. Sheng, K. Ling, Z. Li, and L. Zhao, Effect of steam treatment on catalytic performance of HZSM-5 catalyst for ethanol dehydration to ethylene. Fuel Processing Technology. (110). 2013, 73-78.

[14] A. G. Gayubo, A. Alonso, B. A. T. Aguayo, and J. Bilbao, Selective production of olefins from bioethanol on HZSM5 zeolite catalysts treated with $\mathrm{NaOH}$. Applied Catalysis B: Environmental. (97). 2010, 299-306.

[15] W. Huang, F. Gong, M. Fan, Q. Zhai, C. Hong, and L. Quanxin, Production of light olefins by catalytic conversion of lingo cellulosic biomass with HZSM-5 zeolite impregnated with 6 wt. \% lanthanum. Bioresource Technology. (121). 2012, 248-255.

[16] Huang W, Gong M, Zhai Q, Hong C, Quanxin L. Production of light olefins by catalytic conversion of lignocellulosic biomass with HZSM-5 zeolite impregnated with 6 wt.\% lanthanum. Bio Tech 2012;121:248-255.

http://dx.doi.org/10.1016/j.biortech.2012.05.141 